\title{
The study on Quantitative Evaluation in the Translation Quality Management Based on the House's Translation Quality Assessment Model
}

\author{
Xiyun Liu ${ }^{1}$ Youbin Zhao $^{2}$ \\ ${ }^{1}$ College of Foreign Studies, Jinan University Guangdong 510632, China \\ ${ }^{2}$ School of Translation Studies, Jinan University Guangdong 519070, China
}

\begin{abstract}
Combined with the status quo of the translation quality management in modern translation companies, this paper examines a kind of quantitative evaluation of translation quality based on House's translation quality assessment model and fuzzy mathematics, which overcomes the difficulties found in the translation project and promote the standardization of project management for translation companies.
\end{abstract}

Keywords: Translation quality management; House' model; Quantitative evaluation of translation quality

\section{Introduction}

According to the global language service market report by the Common Sense Advisory, a language service market research firm, global language service market was estimated at $\$ 29$ billion in 2011 and is expected to reach $\$ 47$ in 2015. It is seen that the market for translation has a rapid development. With Chinese economic development increasingly integrated with the global economic development at large, domestic translation industry also has a tremendous development, especially after the 2008 Beijing Olympic Games and the Shanghai World Expo 2010.

However, there are still some problems behind the prosperity such as lack of market admittance standard, lack of language service selection criteria and lack of qualified translators. The greatest problem is that translation quality of some translation companies is too bad to meet the needs of clients. The essential cause is that some translation companies lack a systematic model for evaluating translation quality.

Translation companies always regard quality as their life. Translation quality management is a very important segment in the translation project management for companies. It often relies on translation service quality standards such as Target text quality requirements for translation services-GB/T 19682-2005 enacted by the China National Standardization Management Committee. However, the term of the standard is too descriptive and ambiguous to handle in the modern translation quality management. There is a need to find a more intuitive quantitative evaluation method to assure the quality for the translation companies.

The paper is advanced under this situation. Section 1 discusses the necessity of quantitative evaluation by elaborating the general process of translation quality management. Section 2 explains the possibility of quantitative evaluation by analyzing House's translation quality assessment model. Section 3 applies the fuzzy mathematics to examine a model for translation quality quantitative evaluation. I sincerely hope the thesis can provide some assistance and reference for translation companies to improve the translation quality and maximize their profits.

\section{The necessity of translation quality quantitative evaluation}

Under the background of globalization, commercialization, traditional freelancer model can't meet the needs of the market. Modern translation service must rely on translation companies' commercial operation. They apply the tool of project management (PM) to optimize resource distribution and achieve the balance of quality, cost and schedule.

Found in 1940s, developing in 1980s, project management has become an important branch of management. According to A Guide to the Project Management Body of Knowledge by Project Management Institute, a project is a temporary group activity designed to produce a unique product, service or result. And the project management is the application of knowledge, skills and techniques to execute projects effectively and efficiently. It's a strategic competency for organizations, enabling them to tie project results to business goals — and thus, better compete in their markets. Project management processes fall into five groups: initiating, planning, executing, monitoring \& controlling and closing. Project management knowledge draws on ten areas: integration, scope, time, cost, quality, procurement, human resources, communications, risk management, stakeholder management.

Accordingly, translation project management means the application and implementation of PM in translation market. It includes four progresses (initiating, planning, 
executing and closing) and four key elements (communications, quality, enterprise-owned language tool resources and financing).

For all translation projects, the quality is the key of whether the project can be successfully worked out, so translation quality management becomes the most important progress. It is based on the total quality management, which means a management approach of an organization centered on quality, based on the participation of all its members and aiming at long term success through customer satisfaction and benefits to all members of the organization and society. It includes two main progresses: the production of translation and the quality monitoring of translation.

In the production of translation, translation companies organize experienced and qualified translators to translate and review the task. If necessary, the task is also converted to the original format to check some problems because of converted format. Besides companies can run a Subject Matter Expert Review to check some problems about jargon or run a Stylistic Review to assure a uniform style. And then the task will turn to the quality monitoring of translation. Language Quality Inspection (LQI) is used in this segment, which is a kind of quality control method throughout the whole progress of translation project. Different from the review, it is not modifying the translation but giving a general evaluation of translation quality by sampling. In LQI, companies calculate the translation errors to form a report evaluating the translation quality.

To make cost settlement easier and evaluate the translation quality more intuitive, the result of LQI is always in a quantitative form. However, without taking other elements such as social functions of the texts into account, only the calculation of translation errors can't fully reflect the quality. If such a method is used in LQI, it will have a bad influence on the accuracy of translation quality evaluation. Therefore, building a scientific and convictive quantitative evaluation becomes an urgent issue. From mentioned above, it is seen that the accuracy of LQI in the translation quality management provides the necessity of quantitative evaluation.

\section{The possibility of translation quality quantitative evaluation}

So from which aspects on earth should we assess the translation quality? Juliane House gives the answer.

Translation quality assessment is always a hot topic in translation studies. Many translation theorists have proposed their views about translation standard in China and west. The three-character principle "faithfulness, expressiveness and elegance" by Yan $\mathrm{Fu}$ created a precedent of translation standard research in China. In the 1980s, Professor Gu Zhengkun put forward "Plural Complementarism of Translation Standard". He thought there should be many specific standards to assess different translation. In the meantime, Professor $\mathrm{Xu}$ Yuanchong founded the theory of "Three Principles of Beauty" which means beauty in sense, beauty in sound and beauty in form. In the west, "Critique Productive" by Berman and "Argumentaion-Centered Approach" by Malcolm Williams enriched the theory of translation standard research. However, because of their less feasibility and subjectivity, they can't adapt to the requirement of translation project management and be the theoretical foundation of translation quality quantitative evaluation.

In 1970s, German scholar Juliane House first proposed the concept of translation quality assessment model with the publishing of her works Towards a Model of Translation Quality Assessment and Translation Quality Assessment: A Model Revisited. Her theory is regarded as the first translation quality assessment model with complete theory and practice in the field of the international translation criticism research.

In the House's translation quality assessment model, she thinks the key of assessment is to understand the nature of translation, specifically, the relationship between the original and the translated text. Based on Hallidays' systemic functional linguistics and the theory of discourse analysis, House maintains the nature of translation is the meaning of the linguistic unit remaining unchanged when a kind of language is translated to the other. The meaning refers to the semantic, pragmatic and textual meaning. The semantic meaning reveals the relationship between the linguistic unit and its referent in the real world. The pragmatic meaning connects the linguistic unit with the language speaker in certain circumstances. The textual meaning reflects the semantic relation in the discourse. Therefore, she defines the translation as "the replacement of a text in the source language by a semantically and pragmatically equivalent text in the target language". The assessment of translation quality is to check whether the translated text is equivalent to the original in the semantic, pragmatic and textual meaning.

Given the above analysis, House's model is a kind of scientific and objective appraisal, including both macroscopic and microcosmic standard. Drawing upon House's theory, we can assess the translation from three dimensions of the semantic, pragmatic and textual meaning, which makes it possible to build a model for translation quality quantitative evaluation.

\section{A model for translation quality quantitative evaluation}

Fuzzy mathematics forms a branch of mathematics related to fuzzy set theory and fuzzy logic. It started in 1965 after the publication of Lotfi Asker Zadeh's seminal work Fuzzy sets. The fuzziness is the essential characteristic and the universal phenomenon of the real world in the fuzzy mathematics. The membership function in the fuzzy math is 
used to describe the fuzziness of things. When applied in the translation quality evaluation, it can be used to express the fuzzy evaluation of translation. Firstly, the dimensions of evaluation should be determined. Then we need to make sure the membership function of translation versus the fuzzy sample and get the raw data. After simple calculations, we can get the final result of the translation quality evaluation in a quantitative form. For ease of understanding and operation, the process of the model is simplified as follows:

1. Take the semantic, pragmatic and textual meaning elucidated in section 3 as the dimension of evaluation.

Let $\mathrm{S}$ be the set of dimension.

$$
\mathrm{S}=\left\{\mathrm{s}_{1}, \mathrm{~s}_{2}, \mathrm{~s}_{3}\right\}
$$

$\mathrm{s}_{1}$ : the semantic meaning.

$\mathrm{s}_{2}$ : the pragmatic meaning.

$\mathrm{s}_{3}$ : the textual meaning.

2. According to the different importance of each dimension, add the weight to the model and assign values as follows. Let $\mathrm{W}$ be the set of respective weight.

$$
\begin{gathered}
\mathrm{W}=\left\{\mathrm{w}_{1}, \mathrm{w}_{2}, \mathrm{w}_{3}\right\} \\
\mathrm{w}_{1}=0.5 \quad \mathrm{w}_{2}=0.3 \quad \mathrm{w}_{3}=0.2
\end{gathered}
$$

3. Then the model for translation quality quantitative evaluation can be expressed as follows:

$$
\left\{\mathrm{s}_{1}, \mathrm{~s}_{2}, \mathrm{~s}_{3}\right\} \times\left\{\mathrm{w}_{1}, \mathrm{w}_{2}, \mathrm{w}_{3}\right\}
$$

Make the following rules:

(1)When assessing the translation, take ten sentences as a unit. These ten sentences can be chosen by the equidistant random sampling. For example, a passage with about thirty sentences can be chosen at random from every three sentences. More samples, more accuracy. If the passage is very long, it can be divided into two units or more to assure the accuracy.

Let $\mathrm{Q}$ be the set of quality.

$$
\mathrm{Q}=\left\{\mathrm{q}_{1}, \mathrm{q}_{2}, \cdots, \mathrm{q}_{10}\right\}
$$

$\mathrm{q}_{1}, \mathrm{q}_{2}, \ldots, \mathrm{q}_{10}$ stands for the quality of every chosen sentence. (2)The corresponding quality of each dimension is divided into four levels: $\operatorname{Excellent}(A), \operatorname{Good}(B), \operatorname{Average}(C)$, Poor(D).

(3)Since the semantic meaning determines the correctness of translation, if the semantic meaning is poor, the other two dimensions are marked $\mathrm{D}$.

4. After evaluating a unit, put the result into a matrix. For example:

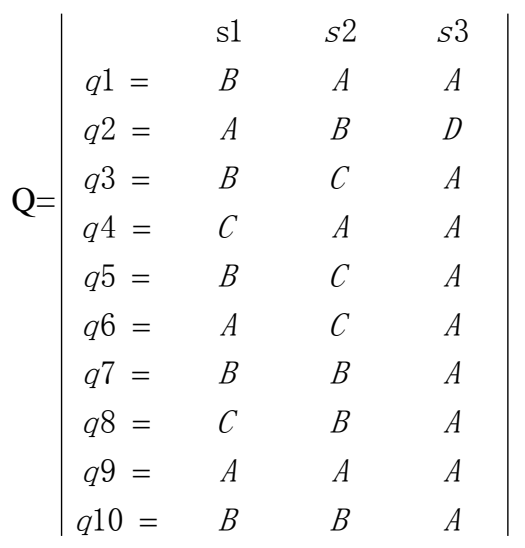

5. Then calculate the number of A,B,C,D for every dimension and put the result into a matrix.

$$
\left|\begin{array}{lllll} 
& A & B & C & D \\
S 1 & 3 & 5 & 2 & 0 \\
S 2 & 3 & 4 & 3 & 0 \\
S 3 & 9 & 0 & 0 & 1
\end{array}\right|
$$

6. Multiply the matrix above by the weight values. The result is:

$$
\begin{aligned}
& A=3 \times 0.5+3 \times 0.3+9 \times 0.2=4.2 \\
& B=5 \times 0.5+4 \times 0.3+0 \times 0.2=3.7 \\
& C=2 \times 0.5+3 \times 0.3+0 \times 0.2=1.9 \\
& D=0 \times 0.5+0 \times 0.3+1 \times 0.2=0.2
\end{aligned}
$$

\section{Analysis:}

\section{$4.2(\mathrm{~A})>3.7(\mathrm{~B})>1.9(\mathrm{C})>0.2(\mathrm{D})$}

Since A gets the maximum (4.2), the passage of this unit can be rated as Excellent. If there are two or more units, we can take the average of every unit's A,B,C,D and then make the final evaluation.

\section{Conclusion}

From the analysis of the above, it is necessary and possible to build a model for translation quality quantitative evaluation.

With the rapid development of the translation industry, we can't simply use a "good" or "bad" to describe the translation quality. The translation quality management in modern companies requires urgently a more accurate method. What's more, we should also find the accurate method needs a solid theory foundation. No matter in a qualitative form or in a quantitative form, translation quality assessment is actually an application of the nature of the translation. House' model points out the direction for the development of the translation quality assessment with its scientificity and systematicness. Besides, fuzzy 
mathematics is a useful tool to help us grasp the fuzzy things in a quantitative form in the real world.

The quantitative evaluation mentioned in this thesis is not absolute. Some parts need further amendments with a great of experiments. The research of translation quality evaluation still has a promising prospect.

\section{Acknowledgments}

This research is sponsored by Jinan University's Scientific Research Creativeness Cultivation Project for Outstanding Undergraduates Recommended for Postgraduate Study.

\section{References}

[1]House Juliane. A Model for Translation Quality Assessment [M]. Germany: Gunter Narr Verlag Tubingen, 1977.
[2]House Juliane. Translation Quality Assessment: A Model Revisited [M]. Germany: Gunter Narr Verlag Tubingen, 1997.

[3]Huawei Wang, Huashu Wang. A Practical Guide to Translation Project Management [M]. Beijing: China Translation \& Publishing Corporation, 2013.

[4]Lei Mu. Further Discussion on Evaluating Translation Quality by Means of Fuzzy Mathematics [J]. Chinese Science \& Technology Translators Journal, 1992(04).

[5]Shengheng Xu. A Mathematical Model for Translation Quality Evaluation [J]. Journal of South China Normal University (Social Sciences Edition), 1987(04).

[6]Shouyi Fan. Fuzzy Mathematics and Translation Quality Evaluation [J]. Chinese Translators Journal, 1987(04). 